\title{
ChRISTIANE LIMBACH
}

Universität Pablo de Olavide, Sevilla

clim@upo.es

ORCID: 0000-0002-3843-3309

\section{ALICE STENDER}

Universität Pablo de Olavide, Sevilla

aste@upo.es

ORCID: 0000-0002-6294-3157

\section{Zur Bewertung des Gesprächsdolmetschens im Hochschulunterricht: Game of Thrones - Wie professionell handelt Missandei?}

\author{
Evaluation of liaison interpreting in university \\ education: Game of Thrones - \\ How professionally does Missandei act?
}

\begin{abstract}
This article aims to present a didactic teaching proposal, which broaches the issue of evaluation of liaison interpreting. An interpreting scene in the popular series Game of Thrones, in whose third season the slave Missandei serves as a negotiating interpreter, will serve as a live demonstration. On the basis of the evaluation criteria elaborated by Kautz (2002), this scene will be examined and analysed in a plenary session, to enable students to form a clear idea about how negotiation interpreting can look in practice, what difficulties can arise, what solutions can be found and what strategies can be adopted. The subsequent discussion serves as a starting point for further reflections about professional ethics, codes of ethics, the neutrality of the interpreter, etc. This teaching proposal has attracted wide interest and its didactic could all be more than achieved.
\end{abstract}

KEYWORDS: liaison interpreting, didactics, evaluation, Missandei.

SCHLÜSSELWORTE: Gesprächsdolmetschen, Didaktik, Bewertung, Missandei. 


\section{EINLEITUNG}

Ziel des Artikels ist es, einen didaktischen Unterrichtsvorschlag für das Gesprächsdolmetschen vorzustellen, im Speziellen soll es hier um die Bewertung von Dolmetschleistungen im Bereich des Gesprächsdolmetschens (oder auch bilateralen Dolmetschens) gehen. Als Anschauungsbeispiel soll uns eine Dolmetschszene der überaus erfolgreichen und weltbekannten Serie Game of Thrones dienen, die auch von vielen Studierenden (und Dozenten) gerne gesehen wird. Die Wahl eines Anschauungsbeispiels aus dieser Serie soll die Studierenden nicht nur motivieren, sondern sie soll als aktuelles Beispiel auch verdeutlichen wie allgegenwärtig das Dolmetschen, und vor allem das Gesprächsdolmetschen, in unserer Kultur (in diesem Fall unserer Unterhaltungskultur) ist.

Unser didaktischer Unterrichtsvorschlag wurde im Kurs „Bilaterales Dolmetschen B/A/B. Alemán" an der Universität Pablo de Olavide in Sevilla (Spanien) im Sommersemester 2017 durchgeführt. Die Studierenden, die dieses Fach im Rahmen ihres Bachelorstudiums „Übersetzen und Dolmetschen" obligatorisch im achten und letzten Semester ihres Studiums belegen, haben bereits im Semester zuvor den Kurs "Dolmetschtechniken B. Deutsch“ absolviert und verfügen somit bereits über Grundkenntnisse im Dolmetschen, sowohl theoretisch als auch praktisch, haben die Notizentechnik erlernt und eine Prüfung im Konsekutivdolmetschen vom Spanischen ins Deutsche abgelegt.

\section{DAS GESPRÄCHSDOLMETSCHEN}

Das Gesprächsdolmetschen ist neben dem Simultan- und Konsekutivdolmetschen eine der drei Dolmetschtechniken ${ }^{1}$ (Collados Aís, Fernández Sánchez \& Stévaux 2001: 48-49), die beim Dolmetschen zum Einsatz kommen können. Im Gegensatz zum Simultan- und Konsekutivdolmetschen, wo fast immer von der Fremdsprache in die Muttersprache gedolmetscht wird, muss beim bilateralen Dolmetschen zusätzlich auch in die Fremdsprache gedolmetscht werden. Es wird also nicht bei einseitigen Gesprächssituationen, wie das hauptsächlich verwendete simultane (also zeitgleiche) oder das seltener verwendete konsekutive (also zeitversetzte) Dolmetschen gebraucht, sondern bei zweiseitigen Gesprächssituationen, also Dialogen zwischen zwei oder mehr Gesprächspartnern. Der Dolmetscher ermöglicht hierbei die Kommunikation zwischen zwei oder mehr Personen, die nicht die gleiche

\footnotetext{
${ }^{1}$ Reinart (2009: 386) verwendet hier den Begriff „Realisierungsmodus“.
} 
Sprache sprechen, sondern zwei verschiedene, und dolmetscht das Gesagte jeweils in die andere Sprache. Festzuhalten ist jedoch ebenfalls, dass das bilaterale Dolmetschen entweder konsekutiv oder simultan durchgeführt werden muss. Da der Dolmetscher beim Gesprächsdolmetschen Teil der Gesprächssituation ist, wird aus praktischen Gründen meist das bilaterale konsekutive Dolmetschen gewählt, wobei der Dolmetscher also jeweils nach einer Äußerungseinheit eines Gesprächsteilnehmers in die andere Sprache dolmetscht.

Das Gesprächsdolmetschen kommt in verschiedenen Bereichen zum Einsatz wie zum Beispiel im Krankenhaus, bei der Polizei, bei Geschäftsverhandlungen, bei Privatgesprächen usw., also immer da, wo Dialoge stattfinden. Die Unterteilung in Dolmetschtechniken auf der einen Seite und Kommunikationsbereiche auf der anderen Seite ist allerdings schwierig, da in der Praxis nicht immer klar unterschieden werden kann (Reinart 2009: 386)².

In unserem Anschauungsbeispiel der Serie Game of Thrones handelt es sich um das Gesprächsdolmetschen bei Geschäftsverhandlungen, auch Verhandlungsdolmetschen genannt.

\section{DIE BEWERTUNG DES GESPRÄCHSDOLMETSCHENS}

Die Bewertung und Qualitätsmessung des Dolmetschens ist nicht einfach und Kriterien müssen darüber hinaus an die verschiedenen Dolmetschtechniken (Simultan-, Konsekutivdolmetschen oder bilaterales Dolmetschen) angepasst werden. Des Weiteren kommt es ebenfalls darauf an, wer die Verdolmetschung bewertet. So formuliert Kautz (2002: 413)

Die Beurteilungskriterien für Dolmetschleistungen sind naturgemäß je nach der Perspektive des Einschätzenden recht divergent. Zum Beispiel wird ein Konferenzveranstalter, -redner bzw. -teilnehmer oder ein Dolmetscher-Kollege die Leistung eines Dolmetschers jeweils zumindest partiell und von der Schwerpunktsetzung her anders bewerten als ein Lehrer (Kautz 2002: 413).

Das Konferenzdolmetschen, und hier vor allem das Simultandolmetschen, hat bisher die meiste Aufmerksamkeit auf sich gezogen. Hier werden vor allem Qualitätsmessungen und -parameter, wie zum Beispiel die vollständige Übertragung des Ausgangstextes in der Zielsprache, die Herstellung der Kommunikation, die Stimme, die Flüssigkeit, usw. in Betracht gezogen (siehe z. B. Collados Aís 2016; Collados Aís, Fernández Sánchez \& Gile 2003; Collados Aís, Pradas Macías, Stévaux \& García Becerra 2007; Collados Aís, Iglesias Fernández, Pradas Macías \& Stévaux 2011). Überlegungen zur

\footnotetext{
2 Für einen kurzen Überblick vgl. Limbach (2018).
} 
Qualität im Allgemeinen (Behr 2013; Gile 2009: 37-48; Pöchhacker 2002; 2004 : 153-158; Prunk 2011) und Überlegungen zur Bewertung des Konsekutivdolmetschens (Kutz 1997), tragen dazu bei, dass bis heute keine einheitlichen Kriterien vorliegen. Viele Forscher arbeiten mit eigenen Kriterien, die sie ihren Bedürfnissen anpassen. $\mathrm{Zu}$ den Qualitätskriterien im bilateralen Dolmetschen ist bis heute deutlich weniger veröffentlicht worden. Festzuhalten bleibt ebenfalls, dass es bisher nur wenige Anhaltspunkte für die Bewertungskriterien im Unterricht (Kautz 2002; Błaszkowska \& Stöckmann 2011) gibt.

Für unsere Zwecke wollen wir uns nun also auf die von Kautz vorgeschlagenen Kriterien und den von ihm entworfenen Fragespiegel stützen (Kautz 2002: 413-4143). Kautz weist darauf hin, dass die Dolmetschleistung nicht abstrakt bewertet werden sollte, sondern dass die Kommunikationssituation und ihre jeweiligen Faktoren berücksichtigt werden müssen (Kautz 2002: 413-414). Es sollte zum Beispiel berücksichtigt werden, ob „der gedolmetschte Text von seiner Schwierigkeit her dem erreichten Ausbildungsniveau des zu evaluierenden Lerners angemessen" war, welche Qualität der Ausgangstext aufweist, ob und wie sich der Lerner "durch entsprechende Recherche auf den Einsatz" vorbereiten konnte, wie die Tonqualität der gehörten Rede war oder die "relevanten äußeren Bedingungen bei live dargebotenen Reden (Sicht des Sprechers? Einwandfreie Mikrofonübertragung?)" waren, ob es sich "bei dem (angenommenen) Adressaten um einen muttersprachlichen Sprecher der Zielsprache oder um eine Person [handelte], die die Zielsprache als Fremdsprache spricht bzw. versteht" (Kautz 2002: 413-414). Des Weiteren weist Kautz (2002: 414) darauf hin, dass ebenfalls in Betracht gezogen werden soll, ob der Lerner in der Lage ist, bestimmte Lösungen und Strategien vor anderen zu verteidigen und zu begründen.

Was nun die konkreten Fragen betrifft, die bei der Bewertung von Dolmetschleistungen eine Rolle spielen, so listet Kautz (2002: 414) folgende Fragen auf:

-Wurde der Dolmetschauftrag insgesamt erfüllt, d.h. war die Kommunikation so einwandfrei, wie es in der gegebenen Situation möglich war? Wurden eventuelle wissensmäßige und/oder sprachliche Defizite des Dolmetschers so kompensiert, dass die Kommunikation dennoch zustande kam?

- Wurde der Sinn des Ausgangstextes vollständig und richtig verstanden und bei der Neuvertextung berücksichtigt? Wurde bei der Redaktion des Zieltextes vor seiner Darbietung weder zu viel weggelassen noch zu viel hinzugefügt?

\footnotetext{
${ }^{3}$ Hierbei muss darauf hingewiesen werden, dass Kautz sich seinerseits auf die Bewertung von Leistungen im Konsekutivdolmetschen an Ausbildungseinrichtungen stützt (Kutz 1997: 252-253).
} 
- Wurde bei der zielsprachlichen Neuvertextung der konkrete Adressat gebührend berücksichtigt? Wurden seine spezifischen Verstehensvoraussetzungen berücksichtigt (z. B. durch Verbalisierung von Präsuppositionen, Verwendung von populärwissenschaftlicher Umschreibungen statt Termini bei einem Nicht-Fachmann)?

- Hat der Lerner die dolmetschtechnischen Fragen gut und sinnvoll gelöst? Hierzu gehören v.a.: Wahl der Dolmetschart; Umgang mit dem Sprechtempo des Redners und mit der Länge der zu dolmetschenden Abschnitte; sinnvolle Speicherung des Gehörten und sicherer Umgang mit den eigenen Notizen; Rückfragen an den Redner; Blickkontakt, Gesten, Gesichtsausdruck; Körperhaltung und sonstige „Kommunikationssignale".

- War die sprachliche Gestaltung des Zieltextes einwandfrei? Hierzu gehörten v.a.: grammatische Korrektheit; Kohärenz und Kohäsion; terminologische Exaktheit; wenige oder keine Selbstkorrekturen; korrekte Wahl des Registers; angemessenes Sprechtempo und damit angemessene Länge der Verdolmetschung; zweckmässige rhetorische Darbietung des Zieltextes (Waren Lautstärke, Pausengestaltung, Intonation, Betonung, Sprechrhythmus adäquat? Gab es Versprecher, Hesitationspausen, „Verlegenheitslachen“, „äh“ u. dgl.?).

- War das Auftreten des Dolmetschers angemessen?

Kautz weist darauf hin, dass die Bewertung von Dolmetschleistung sich als noch schwieriger als die Bewertung von Übersetzungsleistungen gestaltet und die Komponente der Subjektivität nicht vollkommen beseitigt werden kann (Kautz 2002: 415).

Für die Studierenden stellt die Einschätzung der eigenen Dolmetschleistungen ein Hindernis dar und sollte aus diesem Grund in den Dolmetschunterricht mit einbezogen werden. Auch können sich die Studierenden anhand einer Auswertung von Dolmetschleistungen im Unterricht daran orientieren, wie eine Verdolmetschung durchgeführt wird, worauf sie bei ihrem Dolmetscheinsatz achten sollten, wo also die Schwierigkeiten beim konsekutiven bilateralen Gesprächsdolmetschen liegen und wie diese gelöst werden können.

Auch komplexere Überlegungen wie zum Beispiel, wie mit Kulturunterschieden umgegangen werden soll und welchen Stil bzw. welche Rolle der Dolmetscher wählen sollte (neutraler Stil od. vermittelnder Stil, Rolle des Regionalexperten) (vgl. hierzu Kautz 2002: 24; Limbach (im Druck)), sollten im Unterricht besprochen werden. Ebenfalls können Beleidigungen eine Schwierigkeit für den Dolmetscher darstellen, da sich die Frage stellt, ob diese gedolmetscht werden sollen, und wenn ja in welcher Form. Diese Problematik hängt mit der Frage zusammen, wem gegenüber der Dolmet- 
scher verpflichtet ist: nur seinem Auftraggeber gegenüber oder allen am Gespräch teilnehmenden Personen (vgl. hierzu Kautz 2002: 24)? Zu diesen berufsethischen Fragen können ebenfalls verschiedene Ehrenkodexe herangezogen werden, die von Dolmetschervereinigungen (BDÜ, AIIC, usw.) vertreten werden.

Bei unserem didaktischen Unterrichtsvorschlag soll das von uns gewählte Anschauungsbeispiel aus der Serie Game of Thrones den Ausgangspunkt für eine Debatte dieser Schwierigkeiten im Plenum bieten. Wir wollen also zunächst den Fragenspiegel von Kautz abarbeiten bevor wir dann in den nachfolgenden Stunden die oben angesprochenen komplexeren Fragestellungen weiter behandeln.

\section{DIDAKTISCHE ANWENDUNG VON GAME OF THRONES IM UNTERRICHT}

Im Folgenden soll aufgezeigt werden, wie eine Szene aus der Serie Game of Thrones, in der eine Dolmetscherin ein Verhandlungsgespräch zwischen zwei Parteien dolmetscht, im Unterricht als Anschauungsbeispiel genutzt werden kann, um zusammen mit den Studierenden die Dolmetschleistung $\mathrm{zu}$ analysieren und $\mathrm{zu}$ bewerten.

Bereits vor der Bewertung der Szene im Unterricht wurden mit den Studierenden die Bewertungskriterien von Dolmetschleistungen im Bereich des Gesprächsdolmetschens besprochen. Hierbei wurden, wie bereits erwähnt, die Kriterien von Kautz (2002) berücksichtigt. Die Studierenden wissen also, worauf sie achten müssen, um die Verdolmetschung von Missandei bewerten zu können. Um die Bewertung durchführen zu können, sehen die Studierenden die ausgewählte Szene zweimal im Unterricht bevor wir danach dazu übergehen die Verdolmetschung im Plenum zu debattieren. Danach wird den Studierenden ein Arbeitsblatt zur Verfügung gestellt, auf welchem der Fragespiegel von Kautz aufgelistet ist, damit diese Fragen einzeln abgearbeitet werden können. Dies geschieht weiterhin im Plenum. Es soll dabei auch auf folgende Punkte geachtet werden:

- Welche Schwierigkeiten treten bei der Verdolmetschung auf?

- Wie werden diese von Missandei gelöst?

- Welche Strategien wendet Missandei an?

- Welche anderen Lösungsvorschläge gibt es und welche Lösung ist aus welchem Grund adäquat?

-Was kann an der Verdolmetschung von Missandei verbessert werden?

- Gibt es Auffälligkeiten?

- Welche Note bekommt Missandei für ihre Verdolmetschung? 


\subsection{Kurze Inhaltsübersicht über die ausgewählte Szene}

Wir haben für unser Vorhaben eine Szene aus der ersten Folge „Valar Dohaeris" der dritten Staffel von Game of Thrones gewählt. In dieser Folge der Serie kommt Daenerys Targaryen, aus dem Haus Tagaryen, auch genannt die Königin von Meereen, Königin der Andalen, der Ersten Menschen und der Rhoynar, Regentin der sieben Königslande und Beschützerin des Reiches oder Khaleesi in Astapor an. Sie hatte zuvor von ihrem Berater Jorah Mormont gehört, dass es an diesem Ort eine legendäre Elitearmee gibt, die als „Die Unbefleckten” bekannt ist. Da Daenerys den Eisernen Thron erobern möchte, ist sie an dieser Armee überaus interessiert, denn mit ihrer Hilfe könnte sie ihrem Ziel ein Stück näher kommen. Daenerys trifft Kraznys mo Nakloz, Eigentümer und Ausbilder dieser Armee, der ihr diese gerne zeigt und mit ihr über den Preis verhandeln möchte.

Die von uns ausgewählte Szene beginnt mit dem Treffen von Daenerys Targaryen und Kraznys mo Nakloz (Minute 46’05). Bei dem Gespräch zwischen den beiden handelt es sich also um eine Geschäftsbesprechung in einem offiziellen Rahmen. Daenerys Targaryen wird hierbei von ihrem Vertrauten, Ser Jorah Mormont, begleitet. Da die Königin die Sprache der sieben Königslande spricht und Kraznys Hochvalyrisch, stellt Kraznys seine Sklavin Missandei zur Verfügung, um die Verhandlungen zu dolmetschen. Was er nicht weiß, ist dass Daenerys auch seine Sprache spricht und deswegen alles versteht. Da sich dies allerdings erst zu einem späteren Zeitpunkt herausstellt, ist dies für unsere Zwecke nicht relevant. Die Gesprächsinterventionen im Hochvalyrischen sind untertitelt und somit können die Studenten sowohl die Ausgangstextdarbietung, wie auch die Verdolmetschung verstehen.

Unsere Ansicht nacht bietet diese Szene eine perfekte Gelegenheit die verschiedenen Aspekte von Missandeis Dolmetschtätigkeit zu beobachten und sie im Anschluss mit den Studierenden zu analysieren und zu bewerten.

\subsection{Analyse der Verdolmetschung von Missandei}

Die Szene beginnt mit der Ankunft der beiden Parteien auf dem Platz der Strafe. Kraznys erklärt Daenerys was es mit der Armee der Unbefleckten auf sich hat. Während der Erklärungen wendet Missandei die Technik des konsekutiven Gesprächsdolmetschens an; sie wartet also jeweils ab, bis Kraznys eine Pause macht, und dolmetscht dann die folgenden Sequenzen: „Die Unbefleckten stehen schon einen Tag und eine Nacht lang hier. Ohne Essen, ohne Wasser. Sie bleiben stehen, bis sie umfallen. So stark ist ihr Gehorsam." 
Daenerys wendet sich an Kraznys und sagt, wie aus den Untertiteln hervorgeht: „Womöglich wären sie geeignet. Wie bildet Ihr sie aus?” Missandei dolmetscht für Kraznys in das Hochvalyrische und verstößt dabei gegen das Prinzip der Loyalität in der Berufsethik, denn sie sollte, laut Kautz (2002: 24) loyal zu allen Kommunikationsteilnehmern sein und damit "grundsätzlich unparteiisch", dennoch stellt sie sich auf die Seite ihres Herren: "Sie gefallen der Frau aus Westeros, aber sie spricht kein Lob aus, um den Preis niedrig $\mathrm{zu}$ halten. Sie will wissen, wie sie ausgebildet werden". Hier gilt es, zu zwei Dinge zu bemerken: Zum einen, dass generell in der ersten Person gedolmetscht wird, um so "unsichtbar" wie möglich zu sein. Im konsekutiven Gesprächsdolmetschen wird allerdings häufig die dritte Person angewendet, damit, für den Fall, dass es mehr als zwei Gesprächsteilnehmer gibt, keine Missverständnisse auftreten. Hier muss allerdings erwähnt werden, dass dies nicht der Fall ist. Es liegt also kein Grund dafür vor, warum Missandei in der dritten Person dolmetscht. Zum anderen deutet Missandei Daenerys Worte, indem sie eine Kaufabsicht und die Absicht den Preis niedrig halten zu wollen in ihre Worte hinein interpretiert. Die Frage, wie die Soldaten ausgebildet werden, überträgt Missandei stattdessen nicht.

Kraznys ergreift daraufhin das Wort: „Sag ihr, was sie wissen will und mach schnell. Es ist heiß heute". Daraufhin beginnt Missandei mit den Erklärungen: „Mit fünf Jahren beginnt ihre Ausbildung. Jeden Tag von Sonnenaufgang bis Sonnenuntergang, bis sie das Kurzschwert beherrschen, den Schild und die drei Speere. Nur jeder 4. Junge überlebt diese rigorose Ausbildung." Hier wird deutlich, dass Missandei mit der Ausbildung der Soldaten überaus vertraut ist, sie also nicht Kraznys Äußerung in die Sprache der sieben Königreiche dolmetscht, sondern vielmehr seinen direkten Anweisungen an sie selbst folgt und sie nun selbständig die Ausbildung der Soldaten beschreibt. Nach einer kurzen Pause übernimmt Kraznys wieder das Gespräch und Missandei verfällt in das simultane Gesprächsdolmetschen. Sie wechselt also die Dolmetschtechnik: „Ihre Disziplin und Treue sind vollkommen. Sie fürchten nichts". Daraufhin bemerkt Ser Jorah, der Begleiter von Daenerys, dass selbst die tapfersten Männer den Tod fürchten und Missandei erklärt Kraznys: „Der Ritter sagt, selbst tapfere Männer fürchten den Tod". Hier ist nun die Anwendung der dritten Person sowie die Identifikation des Sprechers (Der Ritter) durchaus angebracht, um zu verdeutlichen, dass nicht Daenerys diese Bemerkung gemacht hat, sondern Ser Jorah.

Kraznys wendet sich an die Dolmetscherin und bemerkt abfällig: „Sag dem alten Kerl, er riecht nach Pisse". Woraufhin sie ihren Herrn fragt: „Wirklich, Herr?". Kraznys antwortet verärgert und mit erhobener Stimme: „Nein, nicht wirklich: Bist du ein dummes Mädchen oder eine Ziege, dass du so etwas Dummes fragst". Nach dieser Äußerung von Kraznys, welche 
eine Beleidigung eines seiner Gesprächspartner darstellt, zögert Missandei und anstatt zu dolmetschen fragt sie, ob sie diese Aussage wirklich dolmetschen soll. Sie bemerkt also, dass diese Aussage einem eventuellen Vertragsabschluss abträglich ist. Anstatt die Beleidigung zurückzuziehen, beleidigt Kraznys nun auch Missandei, obwohl er sie in ihrem Zögern seine Worte nicht zu dolmetschen, bestätigt. Missandei ändert daraufhin ihre Körperhaltung, senkt den Blick und sagt dann, anstatt den wirklichen Wortlaut wiederzugeben, „Der gute Herr sagt, die Unbefleckten sind keine Männer. Der Tod bedeutet ihnen gar nichts". Hier fällt auf, dass Missandei wieder in der dritten Person dolmetscht, obwohl klar ist, wer die Worte gesprochen hat. Vielleicht ist dies jedoch ihre Strategie Kraznys als sympathisch darzustellen (Der gute Herr), um den Geschäftsabschluss nicht zu gefährden, sondern im Gegenteil, zu fördern.

Kraznys spricht weiter, „Sag dieser ahnungslosen Hure aus Westeros, sie soll die Augen aufmachen und zusehen". Daraufhin geht er zu einem der Unbefleckten. Kraznys beleidigt nun also auch Daenerys, was Missandei nicht dolmetscht, und im Gegensatz dazu äußerst höflich formuliert: „Er bittet Euch genau zuzusehen, Euer Gnaden”. Dabei wendet sie die Strategie der Kompression an. Kraznys möchte der Königin den absoluten Gehorsam der Soldaten zeigen und schneidet einem von ihnen mit dem Schwert die Brustwarze ab. Daenerys versucht, ihn aufzuhalten und sagt zu Missandei: "Sag dem guten Herrn, es ist nicht nötig“. Kraznys lässt sich aber nicht aufhalten: „Hat sie Angst um seine Nippel? Weiß die dämliche Schlampe nicht, dass wir ihnen die Eier abgeschnitten haben?". Auch nach dieser Beschimpfung ändert Missandei den Gesprächsinhalt, indem sie die Beleidigungen auslässt und deutlich höflicher formuliert: „Der gute Herr weist darauf hin, dass Männer keine Brustwarzen brauchen“. Die darauf folgende Konversation zwischen Kraznys und dem Soldaten (Kraznys zu dem Unbefleckten: „Mit dir bin ich fertig“. Der Unbefleckte: „Dieser hier ist glücklich, Euch gedient zu haben") wird nicht von Missandei gedolmetscht, wodurch Daenerys und Ser Jorah Mormont aus der Unterhaltung ausgeschlossen werden.

Kraznys wendet sich wieder Daenerys zu und beginnt zu sprechen. Missandei wartet ab, um ein vollständiges Äußerungssegment zu hören und dolmetscht dann konsekutiv weiter: „Um seinen Stachelhelm zu erlangen, geht ein Unbefleckter mit einer Silbermark auf den Sklavenmarkt, sucht ein Neugeborenes und tötet es vor den Augen seiner Mutter. Damit, sagt der gute Herr, gewährleisten wir, dass sie keine Schwäche mehr in sich tragen“. Hier fällt zum einen auf, dass Missandei zunächst in der dritten Person dolmetscht (Der gute Herr) und danach in die erste Person Plural verfällt (wir). Daenerys: „Ihr nehmt einer Mutter das Baby aus den Armen, tötet es, wäh- 
rend sie zusieht und bezahlt für ihren Schmerz mit einer Silbermünze?“. Hier gilt es, zu beachten, dass, besonders beim konsekutiven bilateralen Dolmetschen, Gesten, Mimik, Körperhaltung und auch der Gesichtsausdruck eine große Rolle spielen. Missandei interpretiert die Aussage von Daenerys und gibt Kraznys ein Statement über den Gefühlszustand der Mutter der Drachen "Sie ist gekränkt!" bevor sie weiter dolmetscht und dabei fokussiert: „Sie will wissen, ob Ihr eine Mutter für ihr totes Kind mit einer Silbermünze bezahlt". Kraznys spricht wieder, sieht Daenerys herausfordernd in die Augen und sagt: „Was für eine verweichlichte, wimmernde Närrin!“ und Missandei dolmetscht in neutralem Ton und ohne die Beleidigung zu übertragen: „Der gute Herr teilt Euch mit, dass das Geld an den Besitzer des Babys geht. Nicht an die Mutter". Daenerys wechselt das Thema und leitet das Verkaufsgespräch wieder in die beabsichtigten Bahnen: „Wie viele habt Ihr zu verkaufen?“. Kraznys hebt die Hände, zeigt acht Finger und Missandei dolmetscht: „Achttausend“. Sie verbalisiert also Kraznys Geste, die man ohne Kenntnis der Größe seiner Armee nicht unbedingt verstehen kann, da ein Finger hier die Zahl Tausend repräsentieren soll. Um das Gespräch zu beenden, wendet sich Kraznys an Missandei: „Sag der Hure aus Westeros, dass sie Zeit bis morgen hat". Auch in diesem Fall lässt die Dolmetscherin die Beschimpfung aus und reformuliert das Gesagte in neutralem Tonfall: „Der gute Kraznys bittet Euch, um eine schnelle Entscheidung. Viele andere Käufer sind interessiert“. Es bleibt darüber hinaus festzuhalten, dass sie hier wiederum, wie auch bereits zuvor, Kraznys in gutem Licht erscheinen lassen möchte, indem sie ihn als "gut" bezeichnet und in der dritten Person von ihm spricht. Hier endet die von uns ausgesuchte Szene.

\subsection{Bewertung der Verdolmetschung im Unterricht}

Nachdem wir das in Punkt 4 erwähnte Arbeitsblatt an die Studierenden ausgeteilt und den Studierenden Zeit gegeben hatten, Reflexionen darüber anzustellen, stellten wir den Studierenden die Frage, ob die Verdolmetschung von Missandei realistisch sei. Alle Studierenden waren sich einig, dass sie sie als realistisch empfanden, da es, unabhängig von der Ware (in diesem Fall einer Armee), um eine Verhandlung geht. Sie waren allerdings auch der Ansicht, dass die Dolmetscherin sich als Sklavin in einem besonderen "Angestelltenverhältnis" befindet und aus diesem Grund in bestimmten Situationen aus Angst vor Repressalien dazu tendierte, Partei für Kraznys zu ergreifen. Generell waren sie auch der Meinung, dass das Verhalten des Verkäufers nicht dem Standard entspricht, denn eigentlich neigen Verkäufer dazu, die ungeschriebenen Gebote der Höflichkeit einzuhalten, seine Ge- 
sprächspartner also nicht zu beleidigen, damit der Verkauf zustande kommt. Bei der Bewertung der Dolmetscherin Missandei waren die Studierenden sich ebenfalls einig, dass Missandei eine Verständigung zwischen den Parteien ermöglicht und Probleme, wie die Beleidigungen, die Kraznys äußert, im Großen und Ganzen mit Bravour löst: Sie macht kaum Denkpausen, um zu überlegen, wie sie die Unverschämtheiten und Beschimpfungen Kraznys umformulieren kann. Hier handelt es sich auch um eine der größten Schwierigkeiten, die die Studierenden aufgezeigt haben, denn ihrer Meinung nach ist es sehr kompliziert, in einem so kurzen Zeitraum das Äußerungssegment $\mathrm{zu}$ hören, $\mathrm{zu}$ verstehen, $\mathrm{zu}$ analysieren und gleichzeitig eine Strategie $\mathrm{zu}$ entwickeln, welche Segmente übertragen werden können und welche geändert werden müssen (wie z. B. Beschimpfungen), ohne dass der andere Gesprächsteilnehmer bemerkt, dass der Inhalt geändert wurde. Hier entstand ebenfalls eine heiße Debatte darum, ob sich der Dolmetscher nicht neutral verhalten soll und auch die Beschimpfungen dolmetschen soll. Es wurde also diskutiert, wie sich der Dolmetscher in solchen Situationen verhalten kann bzw. zu verhalten hat und welche Anhaltspunkte hierzu Berufsethiken oder Ehrenkodexe bieten. Es handelt sich hier um das größte Dilemma für die Studierenden, da sie, im Gegensatz zu Kraznys, Sympathien für Daenerys hegen, ebenso aber auch aus Berufsethik für die Loyalität beiden Gesprächsteilnehmern gegenüber plädieren. So kamen die Studierenden in der Debatte zu dem Schluss, dass man aus den schon genannten Gründen der Verbindung zwischen Missandei und Kraznys verstehen kann, warum sich Missandei auf die Seite ihres Herren stellt. Einerseits waren sie damit einverstanden, dass die Beschimpfungen nicht gedolmetscht und neu vertextet wurden, da sonst wahrscheinlich das Geschäft nicht zustande gekommen wäre. Andererseits waren die Studierenden auch der Meinung, dass Missandei keine persönlichen Kommentare hätte geben dürfen („Sie gefallen der Frau aus Westeros, aber sie spricht kein Lob aus, um den Preis niedrig zu halten", "Sie ist gekränkt!", "der gute Herr"), da sie damit eindeutig gegen den Ehrenkodex des Dolmetschers verstoßen hat. Bei anderen Voraussetzungen sind also andere Strategien in Betracht zu ziehen.

Was nun andere Anforderungen an die Sprachmittlerin betrifft, so zeigt sie, dass sie über ausreichende Sprachkenntnisse im Hochvalyrischen und der Sprache der sieben Königslande verfügt und es somit nicht zu Verständnisinterferenzen kommt. Sie benutzt keine Notizentechnik, die in einer echten Situation, aufgrund einiger längerer Sprachabschnitte, wahrscheinlich unabdingbar wäre und zeigt somit ein gutes Erinnerungsvermögen. Dabei muss auch bemerkt werden, dass sie mit der Ausbildung der Soldaten bestens vertraut ist und somit auch eventuelle Fachtermini (Stachelhelm, Kurzschwert, etc.) kennt. Aus diesem Grund muss sie, wie Kautz (2002: 323) 
erklärt, nicht ",transkodieren“, „d.h. sie keiner tieferen semantischen Analyse unterziehen" und es kommt nicht zu Hesitationspausen oder Versprechern.

Missandei zeigt eindeutig, dass sie die von Kautz (2002: 324-325) vorgeschlagenen verschiedenen Strategien für die Neuvertextung beherrscht: transkodieren, paraphrasieren, komprimieren, fokussieren, expandieren. Außerdem ist sie in den verschiedenen Dolmetschtechniken bewandert, denn sie wechselt vom konsekutiven Gesprächsdolmetschen in das simultane und zurück in das konsekutive, um den Dialog zwischen den Gesprächsteilnehmern nicht unnötig zu verlängern und als Sprachmittlerin so "unsichtbar" wie möglich zu sein. Die Studierenden waren der Ansicht, dass Missandei die Gesprächssituation, abgesehen von den gelegentlichen Wechseln zwischen der ersten und dritten Person, kohärent, korrekt, ohne Selbstkorrekturen und mit einem angemessenen Register gemeistert hat. Das Ergebnis ist eine sprachtechnisch einwandfreie und professionelle Dolmetschleistung, die von allen Teilnehmern der Gesprächssituation verstanden wurde und die somit den Dolmetschauftrag erfüllt hat. Ebenfalls muss festgehalten werden, dass Missandei das Ziel des Vertragsabschlusses durch das Anwenden bestimmter Strategien und konkreter Lösungen unterstützt und fördert. Was eine konkrete Benotung der von Missandei vorgenommenen Verdolmetschung betrifft, ist diese durchaus im sehr guten Bereich anzusetzen.

\section{FAZIT}

Durch diese didaktische Unterrichtseinheit konnten folgende Ziele erreicht werden:

- die Studierenden haben eine Verdolmetschung bewusst verfolgt und im Plenum zusammen mit dem Lehrenden analysiert,

- die auf Kautz (2002) basierenden Bewertungskriterien wurden angewandt und an einem Beispiel verdeutlicht,

- Schwierigkeiten und deren Lösungen wurden besprochen und ausgewertet,

- alternative Lösungsvorschläge wurden debattiert,

- die Studierenden konnten Probleme erkennen und wurden dafür sensibilisiert, dass es keine offiziellen oder allgemeingültigen Lösungsvorschläge gibt, sondern dass der Dolmetscher selbstständig für ihn vertretbare Lösungen finden muss,

- die Studierenden wurden somit auf Fragen, mit denen sich die Berufsethik und die Ehrenkodexe von Dolmetschervereinigungen beschäftigen, aufmerksam gemacht, um diese Fragen in den nächsten Stunden weiter zu erörtern, 
- die Studierenden wurden darauf hingewiesen, dass sie sich als Dolmetscher positionieren müssen, $d$. h. überlegen müssen, welche bestimmten Strategien und Lösungen sie aus welchen Gründen bevorzugen und wie sie diese vor Kunden und anderen Beteiligten verteidigen können.

Auch durch das ausgewählte Anschauungsbeispiel konnten die Studierenden motiviert werden und bekundeten ihr großes Interesse am Unterricht. Da sie zudem nun eine Verdolmetschung im Detail beobachtet und analysiert hatten, war es ihnen zum einen möglich ihre Angst davor, selbst bilateral zu dolmetschen, abzubauen, sowie zu erkennen, welche Probleme sich ergeben könnten, wie mit ihnen umgegangen werden kann und welche Konsequenzen bestimmte Lösungswege mit sich ziehen. Dadurch wurde sowohl der Dolmetschprozess als auch die Bewertung transparenter. Die Studierenden waren dank dieser Transparenz enthusiastisch selbst in die Rolle des Dolmetschers zu schlüpfen und im Plenum eine Verdolmetschung vorzunehmen, die dann zusammen erneut ausgewertet werden könnte. Dank dieser Bewertungsveranschaulichung konnte also die Bereitschaft der Studierenden, sich selbst aktiv als Dolmetscher in den Unterricht einzubringen und ihre Verdolmetschung auch im Plenum auswerten zu lassen deutlich erhöht werden.

Es bleibt festzuhalten, dass diese Unterrichtseinheit ein voller Erfolg war und sowohl die Studierenden als auch die Dozentinnen äußert motiviert wurden. Wir empfehlen deshalb, diese Unterrichtseinheit zu Beginn des Semesters durchzuführen, da die folgenden Unterrichtseinheiten von dieser Dynamik profitieren und das erlangte Wissen sowie die verringerte Angst selbst vor anderen $\mathrm{zu}$ dolmetschen den nachfolgenden Unterricht positiv beeinflussen.

\section{LITERATURVERZEICHNIS}

Behr, M. (2013). Evaluation und Stimmung. Ein neuer Blick auf Qualität im (Simultan-)Dolmetschen. Berlin: Frank \& Timme.

Błaszkowska, H. / Stöckmann B. (2011). Bewertung von Dolmetschleistungen im Unterricht. Glottodidactica. An International Journal of Applied Linguistics, 38, 73-82. http:// pressto.amu. edu.pl/index.php/gl/article/view/334/241.

Collados Aís, A. (2016). Evaluación de la calidad y entonación del intérprete de simultánea: pautas evaluadoras. MonTI: Monografias de traducción e interpretación, 3, 213-238.

Collados Aís, Á. / Fernández Sánchez, M. / Gile, D. (2003). La evaluación de la calidad en interpretación: investigación. Granada: Comares.

Collados Aís, Á. / Fernández Sánchez, M. / Stévaux, E. (2001). Concepto, técnicas y modalidades de interpretación. In: A. Collados Aís / M. Fernández Sánchez (Hrsg.), Manual de interpretación bilateral (S. 39-60). Granada: Comares. 
Collados Aís, A. / Pradas Macías, M. E. / Stévaux, E. / García Becerra, O. (2007). La evaluación de la calidad en la interpretación simultánea: Parámetros de incidencia. Granada: Comares.

Collados Aís, A. / Iglesias Fernández, E. / Pradas Macías, M. E. / Stévaux, E. (2011). Qualitätsparameter beim Simultandolmetschen. Interdisziplinäre Perspektiven. Tübingen: Narr.

Gile, D. (2009): Basic concepts and models for interpreter and translator training. Amsterdam, Philadelphia: John Benjamins.

Kautz, U. (2002). Handbuch der Didaktik des Übersetzens und Dolmetschens. München: Iudicum.

Kutz, W. (1997). Gut für wen? Zur Bewertung von Konsekutivdolmetschleistungen. In: E. Fleischmann / W. Kutz / P. A. Schmitt (Hrsg.), Translationsdidaktik. Grundlagen der Übersetzungswissenschaft (S. 243-254). Tübingen: Narr.

Limbach, C. (2018). Die Bedeutung der interkulturellen Kompetenz für das Gesprächsdolmetschen. In: B. Beníšková (Hrsg.), Interkulturalität in Sprache, Literatur und Bildung. Interculturality in Language, Literature and Education (S. 51-63). Pardubice: Univerzita Pardubice.

Minahan, D. (2013). Game of Thrones - Valar Dohaeris (1. Folge der 3. Staffel). USA.

Pöchhacker, F. (2002). Researching Interpreting Quality. Models and Methods. In: G. Garzone / M. Viezzi (Hrsg.), Interpreting in the 21st Century. Proceedings of the 1st Conference of Interpreting Studies, Forli, University of Bologna (9-11 November 2000) (S. 95-106). Amsterdam, Philadelphia: Benjamins.

Pöchhacker, F. (2004). Introducing Interpreting Studies. New York: Routledge.

Prunč, E. (2011). Differenzierungs- und Leistungsparameter in Konferenz- und Kommunaldolmetschen. In: C. Kainz / E. Prunč / R. Schögler (Hrsg.), Modelling the Field of Community Interpreting. Questions and Methodology in research and training (S. 21-44). Wien, Berlin: Lit Verlag.

Reinart, S. (2009). Kulturspezifik und Fachübersetzung. Berlin: Frank \& Timme.

Received: 30.06.2018; revised: 17.07.2018 